Journal homepage:http://www.interscience.org.uk

DOI:10.18535/ijahm/v7i6.06

Impact Factor: 4.415

\title{
Haridra (Curcuma Longa Linn.) A Classical Review
}

\author{
Debbarma Promod $^{1}$, Jaiswal Mohanlal. ${ }^{2}$ \\ ${ }^{1}$ P.G. Scholar, Department of DravyagunaVigyan, National Institute of Ayurveda, Jaipur. \\ ${ }^{2}$ Professor, Department of DravyagunaVigyan, National Institute of Ayurveda, Jaipur.
}

\author{
Corresponding Author:- Dr.Promod Debbarma.
}

\begin{abstract}
:
Ayurveda described the use of Haridra (Curcuma longa Linn., Family-Zingibaraceae)for the management of different disease conditions in classical texts. In this article, an attempt has been made to compile various information related to Haridra from three samhitas, more than 6 samgrahagranthas, 12nighantus and other published texts.It is observed that rhizomes of Haridraare used as an ingredient in various formulations, which are effective in more than 40 disease conditions. The main indications include kushtha (skin disease), jwara(fever), kshaya,shvasa(dypnoea), kasa(cough) arsha(piles), vrana(wound), Visa(poisonous), pramehaa (diabetes),pandu (jaundice)etc, rhizomes of Haridra is used in about 13 dosage forms; such as kvatha (decoction), lepa(paste), avaleha (semi-solid preparations),taila (oil), ghrita (fat soluble preparations), vati (pills),churna(powder), rasa (mineral preparations).
\end{abstract}

KEY WORDS:_Haridra, Curcuma longa Linn, Ayurveda, formulations.

\section{INTRODUCTION:}

Ayurveda deals with drugs of plant, animal, metal and mineral origin, where maximum drugs are of plant origin, this drugs are available in the classical texts of Ayurveda named as Vedas (6000 BC), samhitas (1500 BC-600 AD), nighantu and samgrahagranthas (800 AD1900 AD).Haridra one of the classical drug of herbal origin, botanically identified as Curcuma longa Linn. Zingiberaceae family(Purseglove,1972). It is a perennial herb with pulpy,orang,tubours root that grow to about 2 feet in hight and is cultivated in India. The rhizomes of Haridra is pungent,bitter in taste and widely used in Ayurvedic,Unani and Siddha Herbal system for the management of different disease conditions. It is has antimicrobial activity (R.Arutselvi et.al 2012), Antifungal, Antibacterial, Antiinflammatory activity(ManimegalaiV.Et.al 2011).Antioxidant,anticarcinogenic(Majeed et al.,1995 and Miquel et al.,2002). Texts of Ayurveda, systematically recorded the varieties, synonyms, part used, pharmacological properties, actions and indication of Haridra in respective texts. Its use, as an ingredient of compound formulations and as a single drug is recorded in samhitas (treaties) as well as in samgrahagranthas (compendia of Ayurveda). The review summarize the literature review, from various available samhitas (classical texts), nighantus (lexicons), samgrahagranthas (compendia) and some other texts related to prayoga were referred; theSynonyms, properties, actions and various formulations with their adhikara (prime indication) were compiled, critically analyzed and arranged in a systemic manner.

\section{VEDIC PERIOD:- (6000 - 4000 BC.)}

Vedas are considered as the repositories of knowledge since antiguity.It is the oldest excent source of drug whice may be called as oldest drug stock of Ayurveda.InAtharvaVeda Haridra was used for the treatment of kushthaRoga, ${ }^{1}$ commented by SayanacharyaIn Agnipurana (Second-Part) Haridrawas used for the treatment ofArsa, ${ }^{2}$ Prameha, ${ }^{3}$ Vrana ropan, ${ }^{4}$ Kamala, ${ }^{5}$ 
Debbarma Promod ${ }^{1}$, Inte rnational Journal of Ayurvedic\& Herbal Medicine 7(6) Nov.-Dec. 2017 (2963-2972)

SAMHITA KALA: (2000 B.C-1300 A.D)

The Samhitakala is the important landmark in the history of Ayurveda.It is in this kala the Ayurveda came in the glimpses of the common man.In this period various Samhitas and compiled work of various Acharyas have been done and kept forward. These are following- The historical evidence of Haridra can be studied under following division:

Table No- 1 References Charaka Samhita (1000-1500 B.C) ${ }^{6}$

\begin{tabular}{|l|l|l|l|}
\hline Sr.No & Disease & Formulation use & References \\
\hline 1. & Kustha & TiktakhudakadiTailam,Mahakhadiradighrtam & Ch.Ci.7/108.Ch.Ci.7/13 \\
\hline 2. & Shotha & Churna & Ch.Ci.R.11/41. \\
\hline 3. & Udara & Patoladichurna & Ch. Ci.13/119. \\
\hline 4. & Hikka-Shvaasa & Manahshiladighrita & Ch. Ci.17/145. \\
\hline 5. & Arsha & Lepa & Ch. Ci.14/52. \\
\hline 6. & Pandu & Haridraghrita,Punarnavadighrita, & Ch. Ci.16/53.Ch.Ci.16/94. \\
\hline 7. & Kasa & Guruchyaadighrita & Ch.Ci.18/161. \\
\hline 8. & Prameha & Kwath & Ch.Ci.6/31. \\
\hline 9. & Visa & Amrita ghritam. & Ch.Ci.23/246. \\
\hline 10. & Dwibaraniya & Lepa & Ch.Ci.25/114. \\
\hline 11. & Yunivyapad & KasamaryadiGhritam & Ch.Ci.30/53. \\
\hline
\end{tabular}

Table No- 2 References Sushruta Samhita(100 -1500 B.C $)^{7}$

\begin{tabular}{|l|l|l|l|}
\hline Sr.No. & Disease & Formulation & References \\
\hline 1. & Arsha & Lepa, & Su.Ci.6/12. \\
\hline 2. & Bhagandar & Varti,Kalka & Su.Ci.8/30,41. \\
\hline 3. & Kustha & Lepa,Churna,Maha vajraka tail & Su.Ci.9/45,46,56. \\
\hline 4. & Prameha & Swaras, Kwath. & Su.Ci.11/8,9. \\
\hline 5. & Vidradhi & Karanjyaadighrit & Su.Ci.16/17. \\
\hline 6. & Visarpa & Guducyaadighrita & Su.Ci.17/10. \\
\hline 7. & Pandu & Ghrita & Su.Ci.44/15. \\
\hline 8. & Svasa & Swarasa & Su.Ci.51/44. \\
\hline
\end{tabular}


Debbarma Promod ${ }^{1}$, International Journal of Ayurvedic\& Herbal Medicine 7(6) Nov.-Dec. 2017 (2963-2972)

\begin{tabular}{|l|l|l|l|}
\hline 9. & Kasa & Ghrita & Su.Ci.52/35. \\
\hline
\end{tabular}

Table No -3 References Ashtanga Hridya( $7^{\text {Th }}$ Century $)^{8}$

\begin{tabular}{|l|l|l|l|}
\hline Sr.No & Disease & Formulation & References \\
\hline 1. & Sannipaatajajwara & Kwath & A.H.Ci.1/65. \\
\hline 2. & Kasa & VshistaRasayan & A.H.Ci.3/133. \\
\hline 3. & Svas-Hikka & Dhumrpaan & A.H.Ci.4/10. \\
\hline 4. & Trishnaa & Kwath & A.H.Ci.6/73. \\
\hline 5. & Pandu & Ghrita & A.H.Ci.16/36. \\
\hline 6. & Kamala & Anajna & A.H.Ci.16/44. \\
\hline 7. & Kustha & & A.H.Ci.19/42,44,64. \\
\hline 8. & Vatavyadhi & Patoladighrita,Pancatiktaghritaguggulu. & $\begin{array}{l}\text { A.H.U.21/6-8, 57- } \\
58 .\end{array}$ \\
\hline 9. & Mukharoga & Khadiradigutika. & A.H.U.22/71,90-93. \\
\hline 10. & Bhagandar & Madhukadi tail. & A.H.U.27/36. \\
\hline 11. & Kshudraroga & Lepa. & A.H.U.32/22. \\
\hline 12. & Gudaroga & Phalaghrita. & A.H.U.34/64. \\
\hline 13. & Visa & Chandraudayaagada. & A.H.35/26. \\
\hline
\end{tabular}

Table No- 4 Reference sharita Samhita9

\begin{tabular}{|l|l|l|l|}
\hline Sr.No. & Disease & Formulation & References \\
\hline 1. & Kustha & Haridraadi tail & H.S.39/46. \\
\hline 2. & Karnaroga & & H.S.44/11. \\
\hline 3. & Netraroga & Varti & H.S.45/25. \\
\hline
\end{tabular}

Table No 4 References Cakradata $\left(11^{\text {th }} \text { Century }\right)^{10}$

\begin{tabular}{|l|l|l|l|}
\hline Sr.No & Disease & Formulation & References \\
\hline 1. & Swarabhed & AjamodadiChurna & C.D.13/8. \\
\hline 2. & Hikka-swasa & Haridradileha & C.D.16/12. \\
\hline
\end{tabular}


Debbarma Promod ${ }^{1}$, Inte rnational Journal of Ayurvedic\& Herbal Medicine 7(6) Nov.-Dec. 2017 (2963-2972)

\begin{tabular}{|l|l|}
\hline 3. & Kustha \\
\end{tabular}

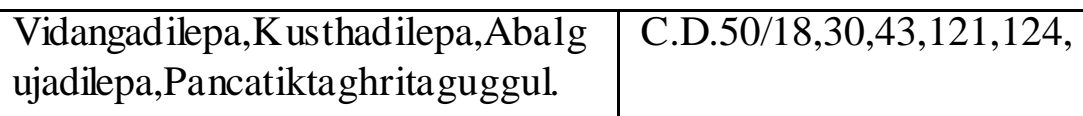

Table No -5 References Sarangdhar ${ }^{11}$

\begin{tabular}{|l|l|l|l|}
\hline Sr.No. & Formulation & Disease & References \\
\hline 1. & Sudharshanchurna & Jwara,Kamala,Pand uroga & S.M.Kh.6/26. \\
\hline 2. & Chandraprabhavati & Prameha,Mutrakrccha & S.M.Kh.7/40. \\
\hline 3. & PunarnvaadiKwath & Shopha. & S.M.Kh.2/120-121. \\
\hline 4. & Brihat-manjishthaadikwath & Sarvakustha. & S.M.Kh.2/138. \\
\hline 5. & Pathyaadisarngakwath & Shamkha,Suryavarta. & S.M.Kh.2/143. \\
\hline
\end{tabular}

Table No- 6 References gadanigraha $\left(12^{\text {th }} \text { Century }\right)^{12}$

\begin{tabular}{|l|l|l|l|}
\hline Sr.No. & Formulation & Diseases & References \\
\hline 1. & Shamkhakaashthilaadi & Shiroroga & G.P-II.S.1/74(1) \\
\hline 2. & Haridraanjanama & Netraroga & G.P-II.S.3/116(1). \\
\hline 3 & Lepa & Upadansh & G.P-II.Sh.8/30(4). \\
\hline 4. & Lepa & Kshudraroga. & G.P-II.Sh.10/31(4). \\
\hline 5. & Lepa & Sarpavish. & G.P-II.Sh.3/11(7). \\
\hline 6. & Lepa & Mushakvish. & G.P-II.Sh.6/2(7). \\
\hline
\end{tabular}

Table No 7 References Bhaisajya Ratnavali $\left(17^{\text {th }} \text { century }\right)^{13}$

\begin{tabular}{|l|l|l|l|}
\hline $\begin{array}{l}\text { Sr. } \\
\text { No }\end{array}$ & Disease & Formulation & References \\
\hline 1. & Jwar & Sudarshanchurna,Jayavati & B.Rt.5/445,534 \\
\hline 2. & Arsha & Suranaadilepa,Vijaychurnam & B.Rt.9/12,40 \\
\hline 3. & Krimi & Krimighatinivatika,Haridrakhanda & B.Rt.11/50,56 \\
\hline 4. & Pandu & PunarnvaadiMandoor,Pandupancanarasa,Haridraghritam & $\begin{array}{l}\text { B.Rt.12/66,100,13 } \\
4\end{array}$ \\
\hline 5. & Hikkaswas & VasadiKwath & B.Rt.16/27 \\
\hline 6. & Swarabheda & Ajamodadichurnam & B.Rt.17/6 \\
\hline 7. & Amavata & Yogarajguggul(Brihat) & B.Rt.29/162 \\
\hline 8. & Prameha & Chandraprabhavati & B.Rt.37/102 \\
\hline 9. & Kustha & $\begin{array}{l}\text { MahamanjisthadiKwath.Mahatiktaghritam,Maricaditaila } \\
\text { m }\end{array}$ & $\begin{array}{l}\text { B.Rt.54/71,244,30 } \\
1\end{array}$ \\
\hline 10. & Mukharoga & Khadiradivati & B.Rt.61/106 \\
\hline 11. & Yonivyapada & Phalaghritam,Soumaghritam & B.Rt.67/74,76 \\
\hline
\end{tabular}


Debbarma Promod ${ }^{1}$, Inte rnational Journal of Ayurvedic\& Herbal Medicine 7(6) Nov.-Dec. 2017 (2963-2972)

\begin{tabular}{|l|l|l|l|}
\hline 12. & Visa & Lepa,Sirisaristam & B.Rt.72/25,38 \\
\hline 13. & Bahumutra & Somnath rasa & B.Rt.86/24 \\
\hline 14. & Sukarameha & TriphaladiKwath & B.Rt.88/15 \\
\hline
\end{tabular}

Table No- 8 References Yogaratnakar ${ }^{14}$

\begin{tabular}{|l|l|l|l|}
\hline $\begin{array}{l}\text { Sr. } \\
\text { No }\end{array}$ & Disease & Formulation & Reference. \\
\hline 1. & Krimi & Churnam & Y.R.P-1.8/25. \\
\hline 2. & Shwas & VasadiKwath,Gudadichurna & Y.R.P-1.14/38,44. \\
\hline 3. & Swarabheda & Ajamodadichurna & Y.R.P-14/18. \\
\hline 4. & Kustha & $\begin{array}{l}\text { NavakKasaya,Sarbangasundarigutika,Vallatak } \\
\text { avaleha, } \\
\text { Mahatiktaghritam,Vajratail,Manjisthadi tail }\end{array}$ & $\begin{array}{l}\text { Y.R.P- } \\
2.55 / 67,84,103,129,141,14 \\
3 .\end{array}$ \\
\hline
\end{tabular}

\section{TABLE NO- 9 REFERENCES BHAVAPRAKASHA ${ }^{15}$}

\begin{tabular}{|l|l|l|l|}
\hline Sr.No & Disease & Formulation & References \\
\hline 1. & Arsha & Punarnvadimandoor, Ksharsutra & B.P.5/30,144. \\
\hline 2. & Shwas & ' & B.P.14/41. \\
\hline 3. & Kustha & $\begin{array}{l}\text { Madhya manjisthadiKwath, } \\
\text { BrihatmanjisthadiKwath, } \\
\text { Mahamaricadi tail }\end{array}$ & B.P. 54/102,104,122. \\
\hline
\end{tabular}

TABLE NO- 10 REFERENCES VANGASEN SAMHITA ${ }^{16}$

\begin{tabular}{|l|l|l|l|}
\hline $\begin{array}{l}\text { Sr. } \\
\text { No }\end{array}$ & Disease & Formulation & References \\
\hline 1. & Kasa & Brihatvasadighrita & V.S.Vol-1.16/162 \\
\hline 2. & Shwas & lepa & V.S.Vol-1.18/47 \\
\hline 4. & Kustha & Navakasaya, Nimbadimahakasaya & V.S.Vol-2. 60/87,88 \\
\hline
\end{tabular}

\section{CLASSICAL CATEGORIZATION AS PER SAMHITA}

CharakaSamhita:-Shirovirechandravya ${ }^{17}$ Lekhaneya mahakasaya ${ }^{18}$,

Kusthaghna ${ }^{19}$ Vishaghna, ${ }^{20}$ Tiktaskandha,${ }^{21}$

SushrutaSamhita:-Haridradi Gana, ${ }^{22}$ Mustadi Gana, ${ }^{23}$ Lakshadi Gana, ${ }^{24}$ Tikta Rasa drabya, ${ }^{25}$

Vagbhata:-Vachaharidradi ${ }^{26}$ MustadiGana ${ }^{27}$ Tikta Rasa dravya, ${ }^{28}$ 
Debbarma Promod ${ }^{1}$, International Journal of Ayurvedic\& Herbal Medicine 7(6) Nov.-Dec. 2017 (2963-2972) REFERENCES NIGHANTU PERIOD

1.Dhanvantari-nighantu(Mahendra bhogika,10 th-13 thCenturyA.D)

Describedvarious 18 synonyms and different Guna karma of Haridra in GuduchyadiVarga(Shloka no55). ${ }^{29}$

2.Shodhalanighantu(12 th Century)-Described 10 synonyms and different qualities of Haridra under GuduchyadiVarga(Shloka no -133,page-12) ${ }^{30}$

3.MadanpalNighantu(14 th Century)

Mentioned 10 synonyms of Haridra and it's different Guna karma in AbhyadiVarga(Shloka no -228.page$187)^{31}$

4.KaiyadevaNighantu(15 th Century A.D.)

Mentioned 20 synonyms and it's different Guna karma in AoushadiVarga(Shloka no 1113-1115). ${ }^{32}$

5.Bhavaprakas Nighantu(15-16 th Century A.D.)

Described 10 synonyms and varieties of Rasa panchakaand Guna karma in HaritakyadiVarga(Shloka no 196-197). ${ }^{33}$

6.RajNighantu(17 th Century A.D.)

Described 30 synonyms it's origin morphological description Guna karma in PippalyadiVarga(Shloka no 196-199). ${ }^{34}$

\section{ShaligramNighantu (19 th Century A.D.)}

Described of 10 synonyms and it'sGuna karma in Haritakyadi Varga. ${ }^{35}$

8.MahaushadiNighantu(20 th Century A.D.)

Described of 14 synonyms and it's different Guna karma in MahaushadiVarga (Shloka no - 117-118). ${ }^{36}$

9.PriyaNighantu(20 thCentury A.D.)

Described of 4 types synonyms and different Guna karma in ShatapuspadiVarga(Shloka no - 170-171) ${ }^{37}$

10.AdarshNighantu (20 th Century A.D.)

Described of Vernacular name,6 types synonyms with each Nirukti and diffarentGuna karma in Adrakadi Varga,Vol-11,page- $556 .^{38}$

11.HrdayadipakNighantu:-Mentioned synonyms and it's Guna karma in EkapadaVarga (Shloka no 118). ${ }^{39}$

TABLE NO- 11 REFERENCES SYNONYMS OF HARIDRAIN NIGHANTU

\begin{tabular}{|l|l|l|l|l|l|l|l|l|l|l|l|l|}
\hline Synonyms & $\mathbf{R}$ & $\mathbf{D}$ & $\mathbf{M}$ & $\mathbf{K}$ & $\mathbf{B}$ & $\mathbf{N}$ & $\mathbf{S U}$ & $\mathbf{S}$ & $\mathbf{P}$ & $\mathbf{H}$ & $\mathbf{M A .}$ & $\mathbf{A}$ \\
& $\mathbf{N}$ & $\mathbf{N}$ & $\mathbf{N}$ & $\mathbf{N}$ & $\mathbf{N}$ & $\mathbf{A}$ & $\mathbf{N}$ & $\mathbf{N}$ & $\mathbf{N}$ & $\mathbf{N}$ & $\mathbf{N}$ & $\mathbf{N}$ \\
& & & & & & & & & & & & \\
\hline Haridra & + & & & & + & + & + & + & + & & + & + \\
\hline Visaghna & + & + & & & & & & & & & & \\
\hline Varabarnini & + & + & + & + & + & & & & & & + & + \\
\hline Subarna & + & & & & & & & & & & & \\
\hline Siva & + & & & & & & & & & & & \\
\hline
\end{tabular}


Debbarma Promod ${ }^{1}$, Inte rnational Journal of Ayurvedic\& Herbal Medicine 7(6) Nov.-Dec. 2017 (2963-2972)

\begin{tabular}{|c|c|c|c|c|c|c|c|c|c|c|c|}
\hline Varnini & + & & & + & & & & & & & \\
\hline Dirgharaga & + & + & & + & & & & & & & \\
\hline Pita & + & + & + & + & + & + & & + & & + & + \\
\hline Varangi & + & & & & & & & & & & \\
\hline Gauri & + & + & + & + & & & & & & & \\
\hline Janistha & + & & & & & & & & & & \\
\hline Para & + & & & & & & & & & & \\
\hline Besava & & & & + & & & & & & & \\
\hline Pindabhara & & & & + & & & & & & & \\
\hline Pitanggi & & & & + & & & & & & & \\
\hline Pinda & & & + & + & & & & & & & \\
\hline Aneshata & & & & & & & + & & & & \\
\hline Gandhapalashika & & & & & & & + & & & & \\
\hline Gharshani & & & & & & & + & & & & \\
\hline Hemaragini & & & & & & & + & & & + & \\
\hline Jvarantika & & & & & & & + & & & & \\
\hline Kaveri & & & & & & & + & & & & \\
\hline Mehaghni & & & & & & & + & & & & \\
\hline Nishakhya & & & & & & + & + & & & & \\
\hline Yybati & & & & & & & & & & + & \\
\hline Varnaatri & + & & & & & & & & & + & \\
\hline Pabira & + & & & & & & + & & & & \\
\hline Harita & + & + & + & & & & & & & & \\
\hline Rajani & + & + & + & + & & & & & + & & \\
\hline Pinga & + & & & & & & & & & & \\
\hline Barnadra & + & & & & & & & & & & \\
\hline Mangalya & + & & & & & & & & & & \\
\hline Lambi & + & & & & & & & & & & \\
\hline Bhadra & + & & & & & & & & & & \\
\hline Shifa & + & & & & & & & & & & \\
\hline Shobhana & + & & & & & & & & & & \\
\hline Shyama & + & & & & & & & & & & \\
\hline Jayanti & + & + & & & & & & & & & \\
\hline Kancani & & & & + & + & & & + & & + & + \\
\hline Nisa & & + & + & + & + & + & & + & & + & + \\
\hline Krimighna & & & & & + & & & & & + & + \\
\hline Haldi & & & & & + & + & & & & + & \\
\hline Yoshipriya & & & & & + & + & & & & + & \\
\hline Hattavilaasini & & & & & + & & & & & + & \\
\hline Varnavati & & + & + & + & & & & & & & \\
\hline Haldika & + & & & & & & & & & & \\
\hline Bharalata & & + & & + & & & & & & & \\
\hline Varnavilasini & & + & & + & & & & & & & \\
\hline Rangini & & + & + & & & & & & & & \\
\hline Romashmulika & & & & + & & & & & & & \\
\hline Pistesta & & & & + & & & & & & & \\
\hline
\end{tabular}

RASAPANCHAK: ${ }^{40} \mathrm{~A}$ drug acts by its potency, which implies all the qualities of drug by which they act,viz. Rasa, Guna, Virya, Vipaka and Prabhava.

Rasa - Tikta, katu. Guna -Ruksha ,Laghu.Vipak -Katu. Veerya - Ushna. Dosakarma -Kapha pita nasaka. 
Debbarma Promod ${ }^{1}$, Inte rnational Journal of Ayurvedic\& Herbal Medicine 7(6) Nov.-Dec. 2017 (2963-2972)

TABLE NO- 12 REFERENCRES RASA PANCHAKAOF HARIDRA IN NIGHANTU

\begin{tabular}{|l|l|l|l|l|l|l|l|l|l|}
\cline { 3 - 11 } \multicolumn{2}{c|}{} & D.N & K.N & M.P & B.N & R.N & P.N & MAN & N.A \\
\hline Rasa & Tikta & + & + & + & + & + & + & + & + \\
\cline { 2 - 11 } & Katu & & + & & + & + & & + & + \\
\hline Vipak & Katu & & + & + & + & + & + & + & \\
\hline Geerya & Ushna & + & + & + & + & + & + & & \\
\hline Dosakarma & Kuksha & + & + & + & + & + & + & + & \\
\hline & Kaphanasaka & & + & & + & + & + & & + \\
\hline & Vitanashak & & + & & + & & + & & \\
\cline { 2 - 11 } & Vatanashak & & & & & & & & + \\
\hline
\end{tabular}

TABLE NO- 13 REFERENCES THERAPEUTIC INDICATION IN NIGHANTU

\begin{tabular}{|l|l|l|l|l|l|l|l|l|}
\hline & Roga & D.N & K.N & R.N & M.N & B.N & MA.N & P.N \\
\hline 1. & Pita roga & + & + & $?$ & + & + & + & + \\
\hline 2. & Kapharoga & + & + & + & + & + & + & - \\
\hline 3. & Vatavikara & + & - & + & - & - & - & - \\
\hline 4. & Raktavikara & - & - & + & + & + & + & - \\
\hline 5. & Kandu & + & - & + & - & - & - & - \\
\hline 6. & Kustha & + & - & - & - & - & - & + \\
\hline 7. & Jwara & + & - & - & - & - & - & - \\
\hline 8. & Daha & + & - & - & + & - & - & - \\
\hline 9. & Pandu & - & + & - & - & + & + & - \\
\hline 10. & Vrana & - & + & + & + & - & + & + \\
\hline 11. & Apchi & - & + & - & + & - & - & - \\
\hline 12. & Prameha & - & + & + & + & + & + & + \\
\hline 13. & Twakdosa & - & + & - & + & + & + & + \\
\hline 14. & Visa & - & + & - & - & + & - & - \\
\hline 15. & Sotha & - & + & - & + & - & + & - \\
\hline
\end{tabular}

R.N-Raj Nighantu. D.N-Dhavantari Nighantu. M.N-Madanpal Nighantu. K.N-Kaiyadeva Nighantu. B.NBhavaprakas Nighantu. N.A-Nighantu Aadarsh. So.N-Shodala Nighantu.S.N- Shaligram Nighantu. P.NPriyavrat Nighantu. H.N-Hridhyadipak Nighantu.MA.N- Madhavadravyaguna Nighantu. A.N-Abhinab Nighantu. 
Debbarma Promod ${ }^{1}$, Inte rnational Journal of Ayurvedic\& Herbal Medicine 7(6) Nov.-Dec. 2017 (2963-2972)

\section{Conclusion:}

The Haridra found in Puran,Vedic ,Samhita period and modern text. It has much valuable properties,can be used to cure many disorders like prameha, kustha,swasa,kasa,jwara, gulma, vrana etc. It is tikta \& katu in rasa,laghu \& ruksha in guna, ushna in veerya and katu in vipaka. The rhizome is part of use and prepare formulations for different diseases conditions. This article collects the huge information of Haridra, will assist to the Ayurvedic industrial people, learners of Ayurveda and the persons interested in Ayurveda with the detailed materials of Haridra.

\section{References:}

1. Atharvedakhandam1,Sutam23,M.2,pub-Dayanandasamsthan ,First Edition,deepmala,sambat 2039.

2. AgnipuranPart-2 ,283.14, by BibekanandaPandey, Ist Eition,Delhi.1997.page-228.

3. Agnipuran Part-2,283.15. , by BibekanandaPandey,IstEition,Delhi.1997.page-246.

4. Agnipuran.Part-2,283.25. , by BibekanandaPandey,Ist Eition,Delhi.1997.page-281.

5. Agnipuran.Part-2,300.34. byBibekanandaPandey,Ist Eition,Delhi.1997.page-310.

6. By Dr.B.K.Twivedi and Dr.PradipkumarGoswami,CharakaSamhita, Chikitsa part-3,pubChaukhambha krishnadas Academy, Varanasi 221001.

7. By KavirajDr.AmbikaduttaShastri, SushrutSamhita, part-1, Edition 2012 and part-2,Uttara Tantra, Edition 2013,pub-Chaukhambha Sanskrit sanshan,Vranasi 221001.

8. Dr.BrahmanandaTripathi, AstangaHrdayam,pub-Chaukhamba Sanskrit pratishthan,Reprint2015,Delhi 110007.

9. ByHarihara Prasad Tripathi, HaritaSamhita, pub-ChaukhambhaKrishnadas Academy,Edition-2009.

10. Cakradatta, Edited and Translated by PriyaVrat Sharma, pub-Chaukhambha ,Vranasi,Edition 2007,

11. ByProf.K.R.SrikanthaMuthy, SarangdharSamhita, pub-ChaukhambhaOrientaliaVaranasi,Edition 2012.

12. ByIndradevaTripathi, Gadanigraha , part-2, Edited by Sri Ganga sahaya Pandey, pub- Chaukhambha Sanskrit Sansthan,Varanasi, Third Edition1994.

13. ,BhaisajyaRatnavali of Govindadas, pub-Chaukhambhaprakashan, Varanasi 221001.

14. ByP.V.Tiwari and Asha Kumari, Gogaratnakar, part-2, Edition 2010, PubChaukhambha Viswabharati, Varanasi 221001.

15. ByProf. K.R..Srikantha Murthy, Bhavaprakasa, Madhyam and Uttar khana.Vol-2, PubChaukhambhaKrishnadas Academy,Edition -Third,2005.

16. BY NirmalSaxena, Bangasen Samhita,Vol-2,Edition 2004, pub- Chaukhambha Sanskrit Series,

17. ByDr.Ramkaran Sharma and Vaidya Bagwan Das,CarakaSamhita.Sutra sthan,Vol-1,Edition 2001, Chap 2,Verse. No 5,Page No 63.

18. By Dr.Ramkaran Sharma and Vaidya Bagwan Das, CarakaSamhita.Sutra sthan,Vol-1,Edition 2001,Chap 4,Verse. No 3,

19. By Dr.Ramkaran Sharma and Vaidya Bagwan Das, CarakaSamhita.Sutra sthan,Vol-1,Edition 2001,Chap 4,Verse. No1 3,

20. By Dr.Ramkaran Sharma and Vaidya Bagwan Das, CarakaSamhita.Sutra sthan, Vol-1,Edition 2001,Chapz

21. By Dr.Ramkaran Sharma and Vaidya Bagwan Das, CarakaSamhita.Sutra sthan,Vol-1,Edition 2001, Viman Sthan Chap 8,Verse. No12 6,

22. By KavirajiDr.AmbikaduttaShastri, prathambhag, SushrutaSamhita Sutrasthan.Eition 2014,pub Chaukhambha Sanskrit Samsthan, Varanasi 221001, Chap 38,V. No 27.

23. By KavirajiDr.AmbikaduttaShastri, prathambhag, SushrutaSamhita Sutra sthan. Eition 2014, pub Chaukhambha Sanskrit Samsthan, Varanasi 221001, Chap 38,V. No 55.

24. By KavirajiDr. AmbikaduttaShastri, prathambhag, SushrutaSamhita Sutra sthan. Eition 2014, pub Chaukhambha Sanskrit Samsthan, Varanasi 221001, Chap 38,V. No 64.

25. By KavirajiDr.AmbikaduttaShastri, prathambhag, SushrutaSamhita Sutra sthan. Eition 2014, pub Chaukhambha Sanskrit Samsthan, Varanasi 221001, Chap 42,V. No 22

26. Bybramananddatripathi, AstangaHridya Sutra sthan,10/29, page 155.

27. A.H.S.15/40. By bramananddatripathi, AstangaHridya Sutra sthan,15/40, page

28. By bramananddatripathi, AstangaHridya Sutra sthan,10/29, page 
Debbarma Promod ${ }^{1}$, International Journal of Ayurvedic\& Herbal Medicine 7(6) Nov.-Dec. 2017 (2963-2972)

29. Dr.amrit Pal Singh, DhanwantariNighantu, GuduchyadiVarga, Puna, 1925. pp.167-168

30. Sodhala, SodhalaNighantu ; Edited by PriyaVrat Sharma, GuduchyadiVarga, Oriental Institute, Baroda, 1st Edition 1978, page-12.

31. MadanpaINighantu,AbhayadiVarga, by Dr. J.L.N. Sastry,publisherschowkhambaorientalia,post boxno-1032,Edition2010.page-187.

32. Kaiyadeva, KaiyadevaNighantu, Edited by P.V.Sharma and Guruprasad Sharma, Aushadhivarga, ChaukambhaOrientatia, Varanasi, 1st Edition, 1979.Shlok No-1113-1115.

33. Bhavaprakasa,BhavaprakasaNighantu,ofSriBhavamisra,HaritakyadiVarga,Commentaryby padmshriProf.K.C.Chunekar,Edited by LateDr.G.S.Pandey,Reprint-2015. ChaukhambhaBharatiAcademy,Varanasi.Shlok No-196-197,page-111-112.

34. IndradevTripathi, Raj Nighantu, Acharya VishwanathDurvedi,PippalyadiVarga,Pub.Krishna Das Academy Varanasi-221001,Shlok No-196-198,page-174.

35. ShaligramVaishya.ShaligramaNighantu,HaritakyadiVarga, Edn1st, KhemrajShrikrishnadasPrakashan, Mumbai, 2002,

36. MahausadhaNighantu,MahaushadiVarga,byP.T.Aryadasa.kumarSingha,INdradeva Tripathi,Chaukha mbhaBharati Academy Varanasi, Reprint2006.Shlok No-117-118.page.55.

37. Sharma PV, PriyaNighantu, ShatapuspadiVarga, ChaukambhaSurabharatiPrakashana, Varanasi, 2004, Shlok No-170-171.page.107.

38. AdarshNighantu,Vol-11,AdrakadiVarga,byBapalalaG. Vaidya.pub.chaukhambhaBharati Academy,Varanasi-221001, Reprint-2016,page-556.

39. HridayadipakaNighantu with Siddhamantra of Vaidyacaryakesava. Ekapadavarga, Edited by Prof.P.V.Sharma. publishers.ChaukambhaAmarabhāratiprakasan, Varanasi -221001.page-9.

40. By.Prof.P.V.Sharma,Dravyaguna-Vijnana,Vol-2,pub-Chaukhambha BharatiAcademy, Varanasi, Reprint 1999. 